\title{
Relación entre la condición física y el hábito de actividad física con la capacidad de atención selectiva en alumnos de enseñanza secundaria
}

\section{Relationship between physical fitness and the physical activity habit with the selective attention capacity in secondary school students}

\section{Relação entre condição física e hábito de atividade física com capacidade de atenção seletiva em estudantes do ensino secundário}

Arboix-Alió, J. ${ }^{1,2}$, Sagristà, F. ${ }^{2}$, Marcaida, S. ${ }^{2}$, Aguilera-Castells, J. ${ }^{1}$, Peralta-Geis, M. ${ }^{1}$, Solà, J. ${ }^{1}$ y Buscà, B. ${ }^{1}$

${ }^{1}$ Facultat de Psicologia, Ciències de l'Educació i de l'Esport Blanquerna, Ramon Llull University (Barcelona, España); ${ }^{2}$ Departamento de Educación Física, Escola Sagrada Familia Urgell (Barcelona, España).

\section{RESUMEN}

El objetivo del presente estudio fue analizar la relación entre las capacidades físicas, el hábito de realizar actividad física y la capacidad de atención selectiva en una muestra de estudiantes de enseñanza secundaria.

La muestra fue configurada por un total de 97 participantes, concretamente 51 estudiantes de género femenino $(n=$ 51) y 46 de género masculino $(n=46)$ de Barcelona (Cataluña) con edades comprendidas entre los 13 y los 15 años $(13,7 \pm 1,17)$. Para analizar la capacidad de atención selectiva se utilizó el Test de Percepción de Semejanzas y Diferencias (CARAS-R). Referente a la condición física, se evaluó la velocidad lineal (30 metros lisos), la fuerza de tren inferior (test de salto horizontal), la agilidad (test de10x5 metros) y la resistencia aeróbica (test de Luc-Léger). Finalmente el hábito de realización de actividad física se calculó con el Cuestionario internacional de actividad física en su formato adaptado para adolescentes (IPAQ-A).

Los resultados mostraron que los participantes con un mayor rendimiento en el test de Luc-Léger, obtuvieron mejores puntuaciones en el test CARAS-R. Asimismo se obtuvo una correlación alta entre los registros del test de Luc-Léger y el test CARAS-R tanto para el género masculino como el femenino y una correlación alta entre el cuestionario IPAQ-A y el test CARAS-R en el género masculino. Dichos hallazgos, ponen de manifiesto la relación entre la atención selectiva y el nivel de condición física en adolescentes. En este sentido, se refuerza la importancia de practicar actividad física a esta edad, sugiriendo que el incremento de la condición física puede tener implicaciones positiva sobre la atención selectiva.

Palabras clave: adolescentes; desarrollo cognitivo; educación secundaria; actividad física.

\section{ABSTRACT}

${ }^{1}$ Correspondence to: Arboix-Alió, Jordi. FPCEE Blanquerna. Universitat Ramon Llull C/Císter 34, 08022. Email: jordiaa1@blanquerna.url.edu. 


\section{Arboix-Alió et al.}

The aim of this study was to analyze the relationship between fitness, selective attention and the physical activity habit in high school students.

The sample was configured by a total of 97 participants, 51 females $(n=51)$ and 46 males $(n=46)$ from Barcelona (Catalonia), aged between 13 and 15 years $(13,7 \pm 1,17)$. To analyze the selective attention capacity, it was used the Perception of Similarities and Differences Test (CARAS-R). Regarding physical fitness, linear velocity (30-meter dash test), lower body strength (board jump test), agility (10x5 meter test) and aerobic capacity (Luc-Léger test) were evaluated. Finally, the physical activity was calculated with the International Physical Activity Questionnaire adapted for adolescents (IPAQ-A).

The results showed that the subjects with higher performance in the Luc-Léger test obtained better scores in the CARAS-R test. A high correlation was also obtained between the records of the Luc-Léger test and the CARAS-R test in both boys and girls and a high correlation between the IPAQ-A questionnaire and the CARAS-R test in boys. These findings highlight the relationship between selective attention and the fitness level in adolescents. In this sense, the importance of practicing physical activity at this age is reinforced, suggesting that the increase of physical condition may have positive implications on selective attention.

Key words: teenagers; cognitive development; secondary education; physical activity.

\section{RESUMO}

O objetivo do presente estudo foi analisar a relação entre habilidades físicas, atenção seletiva e o hábito de praticar atividade física numa amostra de estudantes do ensino médio.

A amostra foi composta por um total de 97 sujeitos, especificamente 51 estudantes do sexo feminino $(n=51)$ e 46 do sexo masculino $(n=46)$ de Barcelona (Catalunha) com idades entre 13 e 15 anos $(13,7 \pm 1,17)$. Para analisar a capacidade de atenção seletiva, foi utilizado o Teste de Percepção de Diferenças e Diferenças (CARAS-R). A condição física foi avaliada através do teste de 30 metros de velocidade suave (velocidade linear), do teste de salto horizontal (força do corpo inferior), do teste de 10x5 metros (agilidade) e do teste de Luc-Léger (resistência). Por fim, o hábito de realizar atividade física foi calculado com o Questionário Internacional de Atividade Física, em formato adaptado para adolescentes (IPAQ-A).

Os resultados mostraram que os sujeitos com maior desempenho no teste Luc-Léger obtiveram melhores pontuações no teste CARAS-R. Da mesma forma, foi obtida uma alta correlação entre os registros do teste Luc-Léger e o teste CARAS-R em meninos e meninas e uma alta correlação entre o questionário IPAQ-A e o teste CARAS-R em meninos. Os resultados revelam a relação entre a atenção seletiva e o nível de aptidão física em adolescentes. Nesse sentido, a importância da prática de atividade física nessa faixa etária é reforçada, sugerindo que o aumento da condição física pode ter implicações positivas na atenção seletiva.

Palavras chave: adolescentes; desenvolvimento cognitivo; Educação secundária; atividade física. 


\section{Actividad física y atención selectiva en adolescentes}

\section{INTRODUCCIÓN}

La falta de actividad física en niños y adolescentes se ha convertido en un problema grave en las sociedades industrializadas en los últimos años. La inactividad física es un factor de riesgo en el desarrollo de diferentes enfermedades como la obesidad y la diabetes (García-Hermoso et al., 2019; Héroux et al., 2013). De hecho, en los últimos años, son varias las investigaciones que han mostrado una disminución considerable en los niveles de aptitud física de los adolescentes (Arboix-Alió et al., 2014; Arboix-Alió, Buscà, et al., 2020; Arboix-Alió, Marcaida, et al., 2020; Ferrari et al., 2015; Tomkinson et al., 2017). Este aspecto es preocupante, puesto que un estilo de vida físicamente activo puede influir positivamente en la estructura y la función cerebral durante la infancia (Asigbee et al., 2018; Conde y Tercedor, 2015; Donnelly et al., 2016).

Además de los beneficios fisiológicos que conlleva la actividad física, también se ha especulado sobre los beneficios cognitivos (de Bruijn et al., 2018; Fernandes et al., 2016; Reigal et al., 2020). Con frecuencia se han destacado los efectos de la actividad física en el proceso de aprendizaje escolar tanto en niños como adolescentes, existiendo en el pasado una tendencia a pensar que el tiempo dedicado a objetivos no académicos tenía un efecto negativo en el rendimiento intelectual del estudiante (Lindner, 2002). En los últimos años, se han realizo diversos estudios analizando la relación existente entre la actividad física desarrollada, el nivel de condición física $\mathrm{y}$ aspectos de nivel cognitivo en niños y adolescentes, encontrado resultados dispares. Aunque hay investigaciones que no muestran relación alguna entre ambos (Ahamed et al., 2007; Daley y Ryan, 2000), autores como Shephard (1997) o Shephard y Trudeau (2005) sugirieron que el hecho de aumentar el tiempo dedicado a la actividad física en la escuela conllevaba beneficios cognitivos como por ejemplo un mejor rendimiento académico de los estudiantes en comparación con aquellos que no realizaban actividad física adicional. Asimismo, también se han encontrado estudios que reportan la relación entre el rendimiento físico y otros aspectos cognitivos como el dominio de las matemáticas (Chaddock-Heyman et al., 2015; Lambourne et al., 2013), la lectura (Chomitz et al., 2009) o la ortografía (Pindus et al., 2016) entre otros. Dicha relación, también ha sido evidente con el procesamiento del lenguaje, la memoria, la velocidad de procesamiento, el funcionamiento ejecutivo o la capacidad de atención (Davis et al., 2011; Donnelly et al., 2016; Li et al., 2014; Páez-Maldonado et al., 2020; Voss et al., 2011). La capacidad de atención, definida como la capacidad de seleccionar los estímulos que son relevantes para un individuo en una situación concreta (Posner y Petersen, 1990), despierta un gran interés tanto entre educadores como en entrenadores deportivos (Domínguez-González et al., 2018; Reloba-Martínez et al., 2017). Se trata de una de las principales funciones cognitivas, directamente relacionada con mecanismos como la percepción, la memoria, el funcionamiento ejecutivo y procesos como el aprendizaje (Rosa-Guillamón, Garcia-Canto, y Carrillo-López, 2019). Por este motivo, en ámbitos como el educativo o el deportivo optimizar el funcionamiento de esta capacidad tiene una gran importancia (Pérez-Lobato et al., 2016).

Las relaciones existentes entre la condición física de los adolescentes o sus hábitos deportivos con aspectos del funcionamiento cognitivo, habitualmente se han centrado en analizar la capacidad de la resistencia aeróbica (Baños et al., 2018; Rosa-Guillamón, GarciaCanto, y Carrillo López, 2019; Rosa-Guillamón, Garcia-Canto, y Carrillo-López, 2019). Sin embargo, otras capacidades como la fuerza, la agilidad o la velocidad lineal han sido menos estudiadas. Ello podría inducir la idea errónea de que dichas capacidades físicas no son tan importantes, cuando estas lo pueden ser tanto como la condición aeróbica para mantener la salud y la función locomotora en general (Amstrong y Welsman, 1997). Además, la gran mayoría de las actividades físicas de niños y adolescentes involucran acciones de alta intensidad que requieren la producción de energía anaeróbica (Tomkinson y Olds, 2008). En este sentido, Bailey et al. (1995) encontraron en un estudio observacional con niños de 6 a 10 años, que éstos participan con mayor frecuencia en actividades intensas y de corta duración que en actividades menos intensas de mayor duración.

Del mismo modo, es poco conocida la relación existente entre la práctica de actividad física, la atención selectiva y el nivel de las distintas capacidades físicas en estudiantes adolescentes de enseñanza secundaria. Por este motivo, el objetivo del presente estudio fue analizar las relaciones entre dichos aspectos en una muestra de estudiantes de secundaria de la ciudad de Barcelona (España). 


\section{MÉTODO}

\section{Participantes}

Un total de 92 estudiantes (51 niñas, 41 niños) participaron en este estudio empírico descriptivo y transversal ex post facto (Thomas et al., 2015). Todos los participantes eran estudiantes de $2^{\circ}$ y $3^{\circ}$ de la Enseñanza Secundaria Obligatoria (ESO) del área metropolitana de Barcelona (Cataluña) con una edad media de 13,7 $\pm 1,17$ años. De acuerdo con el criterio de proximidad domiciliaria por el que se rige el proceso de admisión del alumnado, el nivel socioeconómico de los mismos se consideró en general como medio-alto. Los participantes fueron seleccionados mediante muestreo no probabilístico intencional (muestra de conveniencia).

Se consideraron como criterios de inclusión tener una edad entre 13 y 15 años y asistir con regularidad al colegio ( $>80 \%$ asistencia). Los estudiantes que habían estado lesionados durante más de un mes durante el año académico anterior, y los estudiantes con un alto ausentismo ( $>30 \%$ de ausencias) fueron excluidos del estudio. Todos los padres y tutores legales de todos los estudiantes firmaron un formulario de consentimiento informado para la participación de sus hijos en el estudio después de recibir una explicación clara de los procedimientos experimentales, el protocolo de ejercicio, los beneficios y los posibles riesgos asociados con su participación. Las autoridades educativas y el Comité de Ética en Investigación de la Universidad Ramon Llull en Barcelona aprobaron el desarrollo de este estudio. Todos los protocolos realizados en esta investigación cumplieron con los requisitos especificados en la Declaración de Helsinki (revisada en Fortaleza, Brasil, 2013).

\section{Procedimiento}

\section{Condición física}

La condición física se midió con distintos test de capacidad física pertenecientes a la batería EUROFIT. Cada uno de ellos valoraba una capacidad física distinta.

\section{Resistencia aeróbica}

La resistencia aeróbica se calculó mediante el test de Luc-Léger, más conocido en el ámbito educativo como test de Course Navette (Léger et al., 1988) e incluido en la batería EUROFIT (Research CES,
1993). El test de Luc-Léger es una prueba de resistencia progresiva en la que los sujetos deben correr entre dos líneas paralelas separadas por 20 metros al ritmo de una señal sonora. La velocidad inicial es de $8.5 \mathrm{Km} \cdot \mathrm{h}^{-1}$ y se incrementa en $0.5 \mathrm{Km} \cdot \mathrm{h}^{-}$ ${ }^{1}$ con intervalos de un minuto (paliers). El participante debe pisar detrás de la línea de $20 \mathrm{~m}$ en el momento justo en que se emite la señal sonora o beep. Los participantes continúan hasta que ya no pueden seguir el ritmo de la grabación de audio durante dos rectas consecutivas, momento en el que se registra su puntuación (es decir paliers totales) (Lang, Belanger, et al., 2018). El test de Luc-Léger es considerado la mejor y más popular medida de resistencia cardiorrespiratoria como test de campo en niños y jóvenes debido a su bajo costo, simplicidad y capacidad para evaluar grupos grandes de niños simultáneamente (Castro-Piñero et al., 2010; Lang, Tremblay, et al., 2018). Además, la prueba de LucLéger demuestra una gran fiabilidad test-retest y una validez de moderada a fuerte $\left(r_{\mathrm{p}}=0.78,0.72-0.85\right)$, lo que sugiere que esta prueba es una buena medida de la resistencia aeróbica (Mayorga-Vega et al., 2015). Para emitir la señal sonoro se empleó un teléfono móvil (Xiaomi, Redmi Note 4, Pekín, China) y un altavoz bluetooth (Logitech, UeBoom 2, Pekín, China).

\section{Fuerza de tren inferior}

Para calcular la fuerza de tren inferior se realizó el test de salto horizontal a pies juntos. Cada sujeto realizó dos saltos con un período de descanso de 3 minutos entre salto. El mejor salto de cada sujeto fue el seleccionado. La distancia de salto (metros) se contabilizó con una cinta métrica pegada en el suelo.

\section{Velocidad lineal}

La velocidad lineal se calculó con el test de 30 metros lisos, consistente en correr 30 metros en el mínimo tiempo posible partiendo desde una posición de parado. Las líneas de inicio y finalización estaban claramente marcadas con conos. Cada estudiante completó dos sprints con un período de descanso de 3 minutos entre cada sprint. El tiempo fue registrado con la aplicación MySprint. Esta herramienta de detección ha demostrado una excelente confiabilidad intra e interevaluador para la prueba de sprint de $30 \mathrm{~m}$ (Buscà et al., 2018; Romero-Franco et al., 2017). El tiempo más rápido de los dos sprints se utilizó para un análisis más detallado. 


\section{Actividad física y atención selectiva en adolescentes}

\section{Agilidad}

Para calcular la agilidad se empleó el test de 10x5 metros (Adam, Klissouras, Ravazzolo, Renson, y Tuxworth, 1988). Para ejecutar el test, se colocan conos o líneas de marcaje a cinco metros de distancia entre ellos. El objetivo de esta prueba consiste en recorrer 5 metros 10 veces. De esta forma, el participante correrá hasta la línea de 5 metros, la deberá pisar y volver al punto de inicio (esto 5 veces). Una vez se llegue a la última recta se debe cruzar totalmente la línea, no sólo pisarla, contabilizando el tiempo total mediante un cronómetro. En cada marcador ambos pies deben cruzar completamente la línea (Martínez, 2004). Este test ha demostrado tener una buena fiabilidad test-retest (García-Manso, Navarro, y Ruiz, 1996).

\section{Atención selectiva}

La atención selectiva se midió con el Test de Percepción de Semejanzas y Diferencias (CARAS-R) de Thurstone y Yela (2017). El test CARAS-R evalúa la aptitud para percibir rápido y correctamente semejanzas y diferencias entre patrones de estimulación parcialmente ordenados. Mide las aptitudes perceptivas y atencionales mediante 60 ítems gráficos constituidos por dibujos esquemáticos de caras con trazos elementales. La tarea a realizar consiste en determinar cuál de las tres caras que conforman cada elemento es diferente de las otras dos. Se puede aplicar de forma individual o colectiva en un tiempo de 3 minutos. Al tener un carácter sencillo y lúdico, es una tarea muy bien aceptada por los sujetos evaluados.

Esta versión revisada del test incluye baremos nuevos a partir de una amplia muestra a nivel nacional que supera los 12.000 alumnos. También se han incluido nuevas medidas de rendimiento en la prueba, de manera que además de cuantificar el número de aciertos, se propone cuantificar el número de errores $\mathrm{y}$ poder así extraer adicionalmente información sobre si el patrón de respuesta del alumno evaluado ha sido impulsivo o no (Thurstone y Yela, 2017). Dicha prueba ha mostrado una fiabilidad del 0.95 .

\section{Actividad fisica}

La actividad física realizada por cada estudiante se evaluó con el Cuestionario internacional de actividad física en su formato adaptado para adolescentes:
IPAQ-A (Hagströmer et al., 2008). El IPAQ-A proporciona una evaluación rápida del volumen total de actividad física. El gasto MET-minuto/semana se calculó sumando el tiempo empleado y tipo de actividad realizada para cada participante. Los resultados se transformaron en unidades de índice metabólico (MET). El gasto de energía relativo de cada actividad se tuvo en cuenta multiplicando los minutos semanales por su valor MET siguiendo las pautas expresadas en el manual IPAQ-A.

\section{Análisis estadístico}

El análisis estadístico se realizó utilizando el programa SPSS ${ }^{\circledR}$ (Versión 22 para Windows; SPSS Inc., Chicago, IL, EE.UU.). Se realizó un análisis descriptivo básico calculando los estadísticos de la media, la desviación estándar, el error estándar y el recuento numérico. Se analizó la normalidad de las variables de estudio mediante la prueba de Kolmogorov-Smirnov para más de 50 sujetos y así confirmar el uso de técnicas paramétricas. Tanto la muestra masculina como la femenina se dividieron en dos grupos clasificados según su puntuación en el test CARAS-R. Dicha distribución se realizó en base al percentil 50 de la muestra, distribuyéndose así entre el Grupo-1 (aquellos alumnos con una menor puntuación en el test CARAS-R) y el Grupo-2 (aquellos alumnos con una mayor puntación).

Para evaluar las diferencias en las puntuaciones entre los grupos se utilizó el análisis de la varianza (ANOVA). La estimación del tamaño del efecto se realizó mediante la $d$ de Cohen (Cohen, 1988). Los valores del tamaño del efecto se interretaron siguiendo a Nakagawa y Cuthill (2007) como pequeño $(0.2<$ ES $\leq 0.5)$, moderado $(0.5<\mathrm{ES} \leq 0.8)$ o grande $(\mathrm{ES}>0.8)$. Para analizar las correlaciones entre las medidas de los valores obtenidos entre los test de condición física y los valores de los test IPAQ-A y CARAS-R se utilizó el coeficiente de correlación de Pearson. El grado de correlación fue determinado por la escala de Hopkins (2017), donde $r \leq 0,1$, trivial; $r>0,1-0,3$, pequeño; $r>$ 0,3-0,5, moderado; $r>0,5-0,7$, grande; $r>0,7-0,9$, muy grande; $\mathrm{y} r>0,9-1,0$, extremadamente grande. El nivel de significación se estableció en $p<0,05$. 


\section{RESULTADOS}

En la tabla 1 se presentan los resultados descriptivos de las diferentes variables utilizadas para la realización de este estudio. Los resultados muestran que en las cuatro pruebas de condición física y en el cuestionario IPAQ-A, los chicos $(\mathrm{n}=46)$ obtuvieren unos mejores registros que las chicas $(\mathrm{n}=51)$. En cuanto al Test CARAS-R, los resultados fueron muy similares, sin encontrarse diferencias estadísticamente significativas.

La tabla 2 muestra las variables que hacen referencia a los test de rendimiento físico y al hábito de realizar actividad física. La muestra se encuentra dividida por géneros, y en cada uno de ellos se ha dividido entre dos grupos realizados en función de los valores obtenidos en el test CARAS-R: el Grupo-1, donde se encuentran los participantes que han obtenido una menor puntuación y el Grupo-2, que hace referencia a los que han obtenido una mayor puntuación.
Tabla 1. Análisis descriptivo de los resultados de los distintos test clasificados según género. Datos mostrados en media \pm SD.

\begin{tabular}{ccc}
\hline Test & $\begin{array}{c}\text { Género masculino } \\
(\mathrm{n}=46)\end{array}$ & $\begin{array}{c}\text { Género femenino } \\
(\mathrm{n}=51)\end{array}$ \\
\hline $\mathbf{3 0}$ m (segundos) & $4,94 \pm 0,69$ & $5,43 \pm 0,58$ \\
Detente (metros) & $1,79 \pm 0,29$ & $1,52 \pm 0,23$ \\
Luc-Léger (paliers) & $6,39 \pm 2,6$ & $3,58 \pm 1,43$ \\
10x5 (segundos) & $17,49 \pm 1,73$ & $19,09 \pm 3,29$ \\
IPAQ-A (Mets) & $4564,35 \pm 2380,45$ & $3296,66 \pm 2465,57$ \\
CARAS-R (Aciertos) & $45,17 \pm 8,88$ & $45,43 \pm 8,66$ \\
CARAS-R (Aciertos & $43,37 \pm 9,27$ & $43,69 \pm 8,89$ \\
- Errores) & & \\
\hline
\end{tabular}

En el caso de los participantes de género masculino, se encontraron diferencias estadísticamente significativas entre el Grupo-1 y el Grupo-2 en el Test de $30 \mathrm{~m}(p=0,038)$, en el test de Luc-Léger $(p=$ $0,003)$ y en el test IPAQ-A $(p=0,035)$. En el caso de los participantes de género femenino únicamente se encontró esta diferencia entre grupos en el test de LucLéger $(p=0,040)$.

Tabla 2. Resultados de los test de rendimiento físico según nivel de capacidad de atención.

\begin{tabular}{|c|c|c|c|c|c|c|c|c|}
\hline \multirow{2}{*}{ Test } & \multicolumn{4}{|c|}{ Género masculino } & \multicolumn{4}{|c|}{ Género femenino } \\
\hline & Grupo-1 & Grupo-2 & $p$ valor & ES & Grupo-1 & Grupo-2 & $p$ valor & ES \\
\hline 30 m (s) & $5,15 \pm 0,83$ & $4,72 \pm 0,48$ & $0,038^{*}$ & 0,63 & $5,52 \pm 0,62$ & $5,34 \pm 0,54$ & 0,269 & 0,3 \\
\hline Detente (m) & $1,68 \pm 0,3$ & $1,90 \pm 0,22$ & 0,07 & 0,83 & $1,46 \pm 0,21$ & $1,57 \pm 0,24$ & 0,091 & 0,48 \\
\hline $\begin{array}{l}\text { Luc-Léger } \\
\text { (p) }\end{array}$ & $5,28 \pm 2,78$ & $7,5 \pm 1,91$ & $0,003 *$ & 0,93 & $3,02 \pm 1,13$ & $4,16 \pm 1,51$ & $0,040^{*}$ & 0,85 \\
\hline $10 \times 5(s)$ & $17,9 \pm 2,03$ & $17,07 \pm 1,2$ & 0,104 & 0,49 & $19,97 \pm 1,66$ & $18,17 \pm 4,23$ & 0,050 & 0,56 \\
\hline IPAQ-A (M) & $\begin{array}{c}3829,13 \pm \\
2548,68\end{array}$ & $\begin{array}{c}5299,57 \pm \\
1991,14\end{array}$ & $0,035^{*}$ & 0,64 & $\begin{array}{c}3069,9 \pm 2861 \\
72\end{array}$ & $\begin{array}{c}3532,48 \pm \\
2005,08\end{array}$ & 0,509 & 0,18 \\
\hline
\end{tabular}

s_segundos; m_metros; p_paliers; M_Mets; ES = Effect Size; *_Diferencias significativas entre Grupo-1 y Grupo-2

La tabla 3 muestra una matriz de correlaciones de los test de condición física con el IPAQ-A y el test CARAS-R (Aciertos y Aciertos-Errores). Concretamente son los datos obtenidos en la muestra de género masculino. El único test de condición física que obtuvo una correlación significativa tanto con el CARAS-R como con el test IPAQ-A fue el test de Luc-Léger. Se obtuvo también una correlación significativa entre el test de $30 \mathrm{~m}$ y el test IPAQ-A y entre este segundo y el test CARAS-R.
Por último, en la tabla 4 se muestra la correlación entre los test de condición física y el test CARAS-R y IPAQ-A en la muestra de género femenino. Únicamente se observó una correlación significativa entre el test CARAS-R y el test de Luc-Léger. 


\section{Actividad física y atención selectiva en adolescentes}

Tabla 3. Correlación entre la condición física y los test CARASR y IPAQ-A (chicos).

\begin{tabular}{cccc}
\hline \multirow{2}{*}{ Test } & \multicolumn{2}{c}{ CARAS-R } \\
\cline { 2 - 3 } & Aciertos & $\begin{array}{c}\text { Aciertos - } \\
\text { Errores }\end{array}$ & \\
& & $-0,324$ & $\mathbf{- 0 , 4 5 3 *}$ \\
\hline $\mathbf{3 0} \mathbf{m}$ & $-0,295$ & 0,407 & 0,444 \\
Detente & 0,428 & $\mathbf{0 , 5 1 3 * *}$ & $\mathbf{0 , 7 6 5 * *}$ \\
Luc-Léger & $\mathbf{0 , 5 0 9 * *}$ & $-0,281$ & $-0,378$ \\
10x5 & 0,264 & $\mathbf{0 , 3 2 6 *}$ & 1 \\
IPAQ-A & $\mathbf{0 , 3 0 5 *}$ & \\
\hline
\end{tabular}

* Correlación significativa entre test $(p<0,05) ; * *$ Correlación significativa entre test $(p<0,01)$

Tabla 4. Correlación entre la condición física y los test CARASR y IPAQ-A (chicas).

\begin{tabular}{cccc}
\hline \multirow{2}{*}{ Test } & \multicolumn{2}{c}{ CARAS-R } & \multirow{2}{*}{ IPAQ-A } \\
\cline { 2 - 3 } & Aciertos & $\begin{array}{c}\text { Aciertos - } \\
\text { Errores }\end{array}$ \\
\hline $\mathbf{3 0} \mathbf{~ m}$ & $-0,125$ & $-0,172$ & $-0,041$ \\
Detente & 0,256 & 0,295 & $-0,051$ \\
Luc-Léger & $\mathbf{0 , 5 0 5 * *}$ & $\mathbf{0 , 5 0 5 * *}$ & 0,033 \\
10x5 & $-0,163$ & $-0,204$ & $-0,061$ \\
IPAQ-A & 0,024 & 0,072 & 1 \\
\hline
\end{tabular}

*_Correlación significativa entre test $(p<0,05) ; * *$ Correlación significativa entre test $(p<0,01)$

\section{DISCUSIÓN}

La presente investigación tuvo como objetivo analizar la relación entre las capacidades físicas, el hábito de realizar actividad física y la atención selectiva en alumnos de enseñanza secundaria. El principal hallazgo del estudio fue que el grupo que obtuvo una mejor puntuación en el test CARAS-R obtuvo también un mejor rendimiento en el test de Luc-Léger (tanto para el género masculino como el femenino) y un mejor rendimiento en el test de $30 \mathrm{~m}$ y IPAQ-A (solamente para el género masculino). Asimismo, se obtuvo una correlación grande y muy grande entre el test de Luc-Léger y el test CARAS-R; y el test de LucLéger y el test IPAQ-A respectivamente. Estos resultados siguen la tendencia de investigaciones anteriores donde se muestra una relación positiva entre la práctica de actividad física y el funcionamiento cognitivo tanto en niños como en adolescentes (Chaddock-Heyman et al., 2015; de Bruijn et al., 2018; Pérez-Lobato et al., 2016).

Concretamente en el presente estudio se ha evidenciado que aquellos participantes que obtuvieron unos mejores registros en el test CARAS-R, tuvieron un mejor rendimiento significativo en el test de LucLéger tanto en la muestra masculina como en la femenina. Por lo que respeta a los otros test de condición física, únicamente se obtuvieron diferencias significativas en el test de los 30 metros lisos para la muestra masculina. Estos hallazgos, estarían en consonancia con otros trabajos en los que se sugiere que el rendimiento cardiovascular es la variable que mejor predice el funcionamiento cognitivo en diversas tareas (Buck et al., 2008; Hillman et al., 2009; PérezLobato et al., 2016; Pontifex et al., 2011). De hecho, se ha demostrado que una mejora de la capacidad aeróbica aumenta las monoaminas (dopamina, epinefrina y norepinefrina), lo que resulta en: cambios a corto y largo plazo en la estructura y funcionamiento de las regiones del cerebro que son responsables del aprendizaje (Best, 2010), promueve la angiogénesis y la neurogénesis del hipocampo, que es la parte del cerebro responsable de la memoria (Hassevoort et al., 2016) y tiene el potencial de inducir la vascularización y el crecimiento neural de algunas regiones del cerebro (Donnelly et al., 2016; Esteban-Cornejo et al., 2017).

En relación a las diferencias entre Grupo-1 y Grupo-2 en el test IPAQ-A, se obtuvieron diferencias significativas únicamente en la muestra masculina. El grupo con unos mejores resultados en el test CARAS$\mathrm{R}$ obtuvo una mayor puntuación en el test IPAQ-A mostrando un mayor hábito de realizar actividad física. De este modo, se refuerza otra tendencia ya expuesta en investigaciones anteriores que aquellos adolescentes que practican actividad física regularmente, obtienen una mayor puntuación en los índices generales de atención selectiva y concentración, obteniendo además unos mayores resultados académicos (Buck et al., 2008; Morales et al., 2011). Sin embargo, en el presente estudio no se siguió esta tendencia en la muestra femenina puesto que los resultados del test IPAQ-A no mostraron diferencias significativas entre los dos grupos. Una posible explicación podría encontrarse en el hecho que durante la etapa de la adolescencia hay una gran tasa de abandono de la práctica deportiva 
mayoritariamente en el género femenino (César et al., 2012; Folgar et al., 2013). El hecho de que las estudiantes de secundaria abandonen la práctica deportiva disminuyendo así los registros en el test de hábitos deportivos, puede haber sesgado la muestra igualando a los sujetos que tenían el hábito de realizar actividad física en edades anteriores $\mathrm{y}$ lo han abandonado con aquellos que no tenían dicho hábito deportivo.

Por lo que respecta a la correlación entre los test de condición física y los test CARAS-R e IPAQ-A, cabe destacar las diferencias entre la muestra masculina y femenina. En los chicos, se obtuvo una correlación alta entre el test de Luc-Léger y el test CARAS-R y una correlación moderada entre el test CARAS-R y el test de $30 \mathrm{~m}$. Asimismo, ambos test de condición física obtuvieron correlaciones significativas con el test IPAQ-A (alta y muy alta). En relación al test IPAQ-A $\mathrm{y}$ el test CARAS-R, se obtuvo una correlación moderada. Por el contrario, en la muestra femenina la única correlación significativa que se encontró fue la referente al test de Lúc-Léger con el test CARAS-R. Estos datos reforzarían la importancia que tiene la resistencia cardiovascular en los procesos cognitivos, puesto que la relación existente entre el sistema cardiovascular y el adecuado funcionamiento cerebral es un hecho fisiológico que puede venir determinado por una adecuada oxigenación encefálica, y una mejor perfusión cerebral lo que conlleva un mejor rendimiento cognitivo tal y como ha sido señalado en otras investigaciones. Por otro lado, el hecho que en la muestra femenina el test IPAQ-A no haya correlacionado ni con el test CARAS-R ni con ningún test de condición física puede explicarse por el abandono de la actividad física durante la etapa de la adolescencia, ya mencionado anteriormente.

En cuanto a las posibles limitaciones de la presente investigación, cabe señalar en primer lugar el método de muestreo ya que solo se analizaron los estudiantes de un mismo centro educativo. En futuros estudios, sería interesante aumentar la muestra a varias escuelas y realizar un estudio multicéntrico. En segundo lugar, no se han examinado datos como el peso, la composición corporal o el estado nutricional. Dada la repercusión que estos aspectos pueden tener en los resultados de las pruebas de condición física, sería interesante tenerlo en cuenta para futuras investigaciones y también para analizar su posible relación con los aspectos cognitivos. También, sería interesante efectuar investigaciones con diseños longitudinales y así evaluar la relación de estas variables a lo largo de la etapa madurativa del alumno $\mathrm{y}$ ver si existen periodos dónde la relación entre dichas variables se modifica.

Por otro lado sería adecuado en investigaciones futuras, aparte de valorar los hábitos deportivos de forma cuantitativa (ej. cuantificando los METS consumidos), diferenciar el tipo de práctica deportiva efectuada por el sujeto. De este modo, se podría valorar si existen diferencias en la atención según el tipo de práctica deportiva realizada (deportes individuales, de adversario, colectivos, etc.).

\section{CONCLUSIONES}

El presente estudio muestra que el nivel de condición física, especialmente la capacidad física de la resistencia, está asociada a la capacidad de atención selectiva. De este modo, se contribuye al conocimiento de las relaciones existentes entre estas capacidades físicas y cognitivas sugiriendo que sería beneficioso promover la práctica de la actividad física en la adolescencia.

Teniendo en cuenta que muchos adolescentes no efectúan la actividad física recomendada según la OMS, habría que insistir en la necesidad de valorar adecuadamente el papel de la asignatura de Educación Física en la enseñanza y en los diversos programas de actividad física, planteándose como posible solución aumentar las horas de esta asignatura en el currículo escolar. Los resultados del estudio enfatizan la necesidad de efectuar nuevas investigaciones para determinar la relación causal entre el nivel de condición física y los aspectos cognitivos comentados.

Financiación: Esta investigación ha sido financiada por la Fundació Blanquerna, Universidad Ramon Llull con el número de beca BRB1920-GRIES.

Agradecimientos: Agradecemos a todos los sujetos de estudio por su participación. 


\section{Actividad física y atención selectiva en adolescentes}

\section{BIBLIOGRAFÍA}

1. Adam, C., Klissouras, V., Ravazzolo, M., Renson, R., \& Tuxworth, W. (1988). Eurofit: European Test of Physical Fitness Council of Europe. Committee for the Development of Sport Rome.

2. Ahmed, Y., MacDonald, H., Reed, K., Naylor, P.J., Liu-Ambrose, T., \& McKay, H. (2007). School-Based Physical Activity Does Not Compromise Children's Academic Performance. Medicine \& Science in Sports \& Exercise, 39(2), 371-376.

doi:10.1249/01.mss.0000241654.45500.8e

3. Amstrong, N., \& Welsman, J. (1997). Young people and physical activity. Oxford University Press.

4. Arboix-Alió, J., Buscà, B., Sebastiani, E. M., Aguilera-Castells, J., Marcaida, S., Garcia Eroles, L., \& Sánchez López, M. J. (2020). Temporal trend of cardiorespiratory endurance in urban Catalan high school students over a 20 year period. PeerJ, 8, e10365. doi:10.7717/peerj.10365

5. Arboix-Alió, J., Marcaida, S., \& Puigvert, J. (2020). Evolución de la agilidad y velocidad lineal en una muestra de adolescentes durante los últimos 20 años (1998-2018). SPORT TK-Revista EuroAmericana de Ciencias Del Deporte, 9, 97104. doi:10.6018/sportk.412561

6. Arboix-Alió, J., Puigvert, J., Ramos, E., \& Sebastiani, E. M. (2014). Evolución de la condición física de los alumnos de la escuela maristas la inmaculada de barcelona en los últimos 15 años: un estudio transversal. Revista Horizontes Pedagógicos, 16, 24-33.

7. Asigbee, F. M., Whitney, S. D., \& Peterson, C. E. (2018). The Link Between Nutrition and Physical Activity in Increasing Academic Achievement. Journal of School Health, 88(6), 407-415. doi:10.1111/josh.12625

8. Bailey, R. C., Olson, J., Pepper, S. L., Porszasz, J., Barstow, T. J., \& Cooper, D. M. (1995). The level and tempo of children's physical activities: an observational study. Medicine and Science in Sports and Exercise, 27(7), 1033-1041. http://www.ncbi.nlm.nih.gov/pubmed/7564970

9. Baños, J. C. E., Casas, A. G., Escribano, L. G., Fernández-Marcote, A. R. E., López, P. T., \&
Marcos, L. T. (2018). Influencia de la actividad física y la capacidad aeróbica sobre el rendimiento académico en la adolescencia: una revisión bibliográfica. Journal of Negative and No Positive Results, 3(1), 49-64. doi:10.19230/JONNPR.1614

10. Best, J. R. (2010). Effects of physical activity on children's executive function: Contributions of experimental research on aerobic exercise. Developmental Review, 30(4), 331-351. doi:10.1016/j.dr.2010.08.001

11. Buck, S. M., Hillman, C. H., \& Castelli, D. M. (2008). The Relation of Aerobic Fitness to Stroop Task Performance in Preadolescent Children. Medicine \& Science in Sports \& Exercise, 40(1), 166-172. doi:10.1249/mss.0b013e318159b035

12. Buscà, B., Arboix-Alió, J., Aguilera-Castells, J., Mirada, S., Labrador, J. E., Casadevall, P., \& FortVanmeerhaeghe, A. (2018). Intra- and Inter-Rater Reliability of Mysprint App for Measuring Speed Performance in 30 Meters. Intra- and Inter-Rater Reliability of Mysprint App for Measuring Speed Performance in 30 Meters, 23th Annual Congress of the European College of Sport Science, At Dublin, 237-238.

13. Castro-Piñero, J., Artero, E. G., Espana-Romero, V., Ortega, F. B., Sjostrom, M., Suni, J., \& Ruiz, J. R. (2010). Criterion-related validity of fieldbased fitness tests in youth: a systematic review. British Journal of Sports Medicine, 44(13), 934943. doi:10.1136/bjsm.2009.058321

14. César, A., Baena, M., Chillón, P., Martín-matillas, M., López, I. P., Castillo, R., Vicente-rodríguez, G., Casajús, J. A., Álvarez-Granda, L., Romero, C., \& Tercedor, P. y M. D.-F. (2012). Motivos de abandono y no práctica de actividad físicodeportiva en adolescentes españoles: estudio Avena. Cuadernos de Psicologia Del Deporte., $12,45-54$.

15. Chaddock-Heyman, L., Erickson, K. I., Kienzler, C., King, M., Pontifex, M. B., Raine, L. B., Hillman, C. H., \& Kramer, A. F. (2015). The Role of Aerobic Fitness in Cortical Thickness and Mathematics Achievement in Preadolescent Children. PLOS ONE, 10(8), e0134115. doi:10.1371/journal.pone.0134115 
16. Chomitz, V. R., Slining, M. M., McGowan, R. J., Mitchell, S. E., Dawson, G. F., \& Hacker, K. A. (2009). Is There a Relationship Between Physical Fitness and Academic Achievement? Positive Results From Public School Children in the Northeastern United States. Journal of School Health, 79(1), 30-37. doi:10.1111/j.17461561.2008.00371.x

17. Cohen, J. (1988). Statistical power analysis for behavioural science. NJ: Lawrence Erlbaum.

18. Conde, M. A., \& Tercedor, P. (2015). La actividad física, la educación física y la condición física pueden estar relacionadas con el rendimiento académico y cognitivo en jóvenes. Revisión sistemática. Archivos de Medicina Del Deporte, 32(2), 100-109.

19. Daley, A. J., \& Ryan, J. (2000). Academic Performance and Participation in Physical Activity by Secondary School Adolescents. Perceptual and Motor Skills, 91(2), 531-534. doi:10.2466/pms.2000.91.2.531

20. Davis, C. L., Tomporowski, P. D., McDowell, J. E., Austin, B. P., Miller, P. H., Yanasak, N. E., Allison, J. D., \& Naglieri, J. A. (2011). Exercise improves executive function and achievement and alters brain activation in overweight children: A randomized, controlled trial. Health Psychology, 30(1), 91-98. doi:10.1037/a0021766

21. de Bruijn, A. G. M., Hartman, E., Kostons, D., Visscher, C., \& Bosker, R. J. (2018). Exploring the relations among physical fitness, executive functioning, and low academic achievement. Journal of Experimental Child Psychology, 167, 204-221. doi:10.1016/j.jecp.2017.10.010

22. Domínguez-González, F., Moral-Campillo, L., Reigal, R. ., \& Hernández-Mendo, A. (2018). Condición física $\mathrm{y}$ atención selectiva en una muestra preadolescente. Cuadernos de Psicologia Del Deporte, 18(2), 33-42. http://revistas.um.es/cpd

23. Donnelly, J. E., Hillman, C. H., Castelli, D., Etnier, J. L., Lee, S., Tomporowski, P., Lambourne, K., \& Szabo-Reed, A. N. (2016). Physical Activity, Fitness, Cognitive Function, and Academic Achievement in Children. Medicine \& Science in Sports \& Exercise, 48(6),
$1197-1222$.

doi:10.1249/MSS.0000000000000901

24. Dwyer, T., Sallis, J. F., Blizzard, L., Lazarus, R., \& Dean, K. (2001). Relation of Academic Performance to Physical Activity and Fitness in Children. Pediatric Exercise Science, 13(3), 225237. doi:10.1123/pes.13.3.225

25. Esteban-Cornejo, I., Cadenas-Sanchez, C., Contreras-Rodriguez, O., Verdejo-Roman, J., Mora-Gonzalez, J., Migueles, J. H., Henriksson, P., Davis, C. L., Verdejo-Garcia, A., Catena, A., \& Ortega, F. B. (2017). A whole brain volumetric approach in overweight/obese children: Examining the association with different physical fitness components and academic performance. The ActiveBrains project. NeuroImage, 159, 346354. doi:10.1016/j.neuroimage.2017.08.011

26. Fernandes, V. R., Ribeiro, M. L. S., Melo, T., Maciel-Pinheiro, P. de T., Guimarães, T. T., Araújo, N. B., Ribeiro, S., \& Deslandes, A. C. (2016). Motor coordination correlates with academic achievement and cognitive function in children. Frontiers in Psychology, 7(MAR), 1-8. doi:10.3389/fpsyg.2016.00318

27. Ferrari, G. L. D. M., Matsudo, V. K. R., \& Fisberg, M. (2015). Changes in physical fitness and nutritional status of schoolchildren in a period of 30 years (1980-2010). Revista Paulista de Pediatria, 33(4), 415-422. doi:10.1016/j.rppede.2015.03.001

28. Folgar, M. I., Juan, F. R., \& Boubeta, A. R. (2013). Variables predictoras del abandono de la práctica físico-deportiva en adolescentes. Cultura, Ciencia y Deporte, 8, 93-102. doi:10.12800/ccd.v8i

29. García-Hermoso, A., Ramírez-Vélez, R., \& Saavedra, J. M. (2019). Exercise, health outcomes, and pædiatric obesity: A systematic review of meta-analyses. Journal of Science and Medicine in Sport, 22(1), 76-84. doi:10.1016/j.jsams.2018.07.006

30. García Manso, J. M., Navarro, M., \& Ruiz, J. A. (1996). Pruebas para la valoración de la capacidad motriz en el deporte. GYMNOS.

31. Hagströmer, M., Bergman, P., De Bourdeaudhuij, I., Ortega, F. B., Ruiz, J. R., Manios, Y., ReyLópez, J. P., Phillipp, K., von Berlepsch, J., \& Sjöström, M. (2008). Concurrent validity of a 


\section{Actividad física y atención selectiva en adolescentes}

modified version of the International Physical Activity Questionnaire (IPAQ-A) in European adolescents: The HELENA Study. International Journal of Obesity, 32(S5), S42-S48. doi:10.1038/ijo.2008.182

32. Hassevoort, K. M., Khan, N. A., Hillman, C. H., \& Cohen, N. J. (2016). Childhood Markers of Health Behavior Relate to Hippocampal Health, Memory, and Academic Performance. Mind, Brain, and Education, 10(3), 162-170. doi: $10.1111 / \mathrm{mbe} .12108$

33. Héroux, M., Onywera, V., Tremblay, M. S., Adamo, K. B., Lopez Taylor, J., Jáuregui Ulloa, E., \& Janssen, I. (2013). The Relation between Aerobic Fitness, Muscular Fitness, and Obesity in Children from Three Countries at Different Stages of the Physical Activity Transition. ISRN Obesity, 2013, 1-10. doi:10.1155/2013/134835

34. Hillman, C. H., Buck, S. M., Themanson, J. R., Pontifex, M. B., \& Castelli, D. M. (2009). Aerobic fitness and cognitive development: Event-related brain potential and task performance indices of executive control in preadolescent children. Developmental Psychology, 45(1), 114-129. doi:10.1037/a0014437

35. Lambourne, K., Hansen, D. M., Szabo, A. N., Lee, J., Herrmann, S. D., \& Donnelly, J. E. (2013). Indirect and direct relations between aerobic fitness, physical activity, and academic achievement in elementary school students. Mental Health and Physical Activity, 6(3), 165171. doi:10.1016/j.mhpa.2013.06.002

36. Lang, J. J., Belanger, K., Poitras, V., Janssen, I., Tomkinson, G. R., \& Tremblay, M. S. (2018). Systematic review of the relationship between 20 $\mathrm{m}$ shuttle run performance and health indicators among children and youth. Journal of Science and Medicine in Sport, 21(4), 383-397. doi:10.1016/j.jsams.2017.08.002

37. Lang, J. J., Tremblay, M. S., Léger, L., Olds, T., \& Tomkinson, G. R. (2018). International variability in $20 \mathrm{~m}$ shuttle run performance in children and youth: who are the fittest from a 50country comparison? A systematic literature review with pooling of aggregate results. British Journal of Sports Medicine, 52(4), 276. doi:10.1136/bjsports-2016-096224
38. Léger, L. A., Mercier, D., Gadoury, C., \& Lambert, J. (1988). The multistage 20 metre shuttle run test for aerobic fitness. Journal of Sports Sciences, 6(2), 93-101. doi:10.1080/02640418808729800

39. Li, L., Men, W.-W., Chang, Y.-K., Fan, M.-X., Ji, L., \& Wei, G.-X. (2014). Acute Aerobic Exercise Increases Cortical Activity during Working Memory: A Functional MRI Study in Female College Students. PLoS ONE, 9(6), e99222. doi:10.1371/journal.pone.0099222

40. Lindner, K. (2002). The physical activity participation-academic performance rela- tionship revisited: perceived and actual performance and the effect of banding (academic tracking). Pediatric Exercise Science, 14, 155-169.

41. Martínez, E. (2004). Aplicación de la prueba de velocidad 10 × 5 metros, sprint de 20 metros y tapping-test con los brazos. Resultados y análisis estadístico en educación secundaria. Revista Internacional de Medicina y Ciencias de La Actividad Física y El Deporte, 4, 1-17.

42. Mayorga-Vega, D., Aguilar-Soto, P., \& Viciana, J. (2015). Criterion-Related Validity of the 20-M Shuttle Run Test for Estimating Cardiorespiratory Fitness: A Meta-Analysis. Journal of Sports Science \& Medicine, 14(3), 536-547. http://www.ncbi.nlm.nih.gov/pubmed/26336340

43. Morales, J., Gomis, M., Pellicer-Chenoll, M., García-Massó, X., Gómez, A., \& González, L.-M. (2011). Relation between Physical Activity and Academic Performance in 3rd-Year Secondary Education Students. Perceptual and Motor Skills, 113(2), 539-546. doi:10.2466/06.11.13.PMS.113.5.539-546

44. Nakagawa, S., y Cuthill, I. C. (2007). Effect size, confidence interval and statistical significance: a practical guide for biologists. Biological Reviews, 82(4), 591-605. doi:10.1111/j.1469185X.2007.00027.x

45. Páez-Maldonado, J. A., Reigal, R. E., MorilloBaro, J. P., Carrasco-Beltrán, H., HernándezMendo, A., \& Morales-Sánchez, V. (2020). Physical Fitness, Selective Attention and Academic Performance in a Pre-Adolescent Sample. International Journal of Environmental 
Research and Public Health, 17(17), 6216. doi:10.3390/ijerph17176216

46. Pérez-Lobato, R., Reigal, R. E., \& HernándezMendo, A. (2016). Relaciones entre la práctica física, condición física y atención en una muestra adolescente. Revista de Psicologia Del Deporte, 25(1), 179-186.

47. Pindus, D. M., Drollette, E. S., Scudder, M. R., Khan, N. A., Raine, L. B., Sherar, L. B., Esliger, D. W., Kramer, A. F., \& Hillman, C. H. (2016). Moderate-to-Vigorous Physical Activity, Indices of Cognitive Control, and Academic Achievement in Preadolescents. The Journal of Pediatrics, 173, 136-142. doi:10.1016/j.jpeds.2016.02.045

48. Pontifex, M. B., Raine, L. B., Johnson, C. R., Chaddock, L., Voss, M. W., Cohen, N. J., Kramer, A. F., \& Hillman, C. H. (2011). Cardiorespiratory fitness and the flexible modulation of cognitive control in preadolescent children. Journal of Cognitive Neuroscience, 23(6), 1332-1345. doi:10.1162/jocn.2010.21528

49. Posner, M. I., \& Petersen, S. E. (1990). The Attention System of the Human Brain. Annual Review of Neuroscience, 13(1), 25-42. doi:10.1146/annurev.ne.13.030190.000325

50. Reigal, R. E., Moral-Campillo, L., Mier, R. J. De, Morillo-Baro, J. P., Morales-Sánchez, V., Pastrana, J. L., \& Hernández-Mendo, A. (2020). Physical Fitness Level Is Related to Attention and Concentration in Adolescents. Frontiers in Psychology, 11(February), 1-9. doi:10.3389/fpsyg.2020.00110

51. Reloba-Martínez, S., Reigal-Garrido, R. E., Hernández-Mendo, A., Martínez-López, E. J., Martín-Tamayo, I., \& Chirosa-Ríos, L. J. (2017). Efectos del ejercicio físico extracurricular vigoroso sobre la atención de escolares. Revista de Psicologia Del Deporte, 26(2), 29-36.

52. Research CES. (1993). EUROFIT: Handbook for the EUROFIT Tests of Physical Fitness (2nd ed). Committee of Experts on Sports Research.

53. Romero-Franco, N., Jiménez-Reyes, P., CastañoZambudio, A., Capelo-Ramírez, F., RodríguezJuan, J. J., González-Hernández, J., ToscanoBendala, F. J., Cuadrado-Peñafiel, V., \& Balsalobre-Fernández, C. (2017). Sprint performance and mechanical outputs computed with an iPhone app: Comparison with existing reference methods. European Journal of Sport Science, $\quad$ 17(4), 386-392. doi:10.1080/17461391.2016.1249031

54. Rosa-Guillamón, A., Garcia-Canto, E., \& Carrillo-López, P. J. (2019). Relación entre capacidad aeróbica y el nivel de atención en escolares de primaria. Retos. Nuevas Tendencias En Educación Física, Deporte y Recreación, 35, 36. www.retos.org

55. Rosa-Guillamón, A., Garcia-Canto, E., \& Carrillo López, P. J. (2019). Capacidad aeróbica y rendimiento académico en escolares de educación primaria. Retos. Nuevas Tendencias En Educación Física, Deporte y Recreación, 35. www.retos.org

56. Shephard, R. J. (1997). Curricular Physical Activity and Academic Performance. Pediatric Exercise Science, 9(2), 113-126. doi:10.1123/pes.9.2.113

57. Shephard, R. J., \& Trudeau, F. (2005). Lessons Learned from the Trois-Rivières Physical Education Study: A Retrospective. Pediatric Exercise Science, 17(2), 112-123. doi:10.1123/pes.17.2.112

58. Thomas, J. R., Nelson, J. K., \& Silverman, S. (2015). Research Methods in Physical Activity. Human Kinetics.

59. Thurstone, L. L. y Yela, M. (2012). Caras-R: test de percepción de diferencias-revisado: manual. Madrid: Tea.

60. Tomkinson, G. R., Lang, J. J., \& Tremblay, M. S. (2017). Temporal trends in the cardiorespiratory fitness of children and adolescents representing 19 high-income and upper middle-income countries between 1981 and 2014. British Journal of Sports Medicine, bjsports-2017-097982. doi:10.1136/bjsports-2017-097982

61. Tomkinson, G. R., R., Olds, T., \& S. (2013). Field tests of fitness (Vol. 1, Issue December 2015). Oxford University Press. doi:10.1093/med/9780199232482.003.0009

62. Voss, M. W., Chaddock, L., Kim, J. S., VanPatter, M., Pontifex, M. B., Raine, L. B., Cohen, N. J., Hillman, C. H., \& Kramer, A. F. (2011). Aerobic fitness is associated with greater efficiency of the network underlying cognitive control in 
Actividad física y atención selectiva en adolescentes

preadolescent children. Neuroscience, 199, 166-

176. doi:10.1016/j.neuroscience.2011.10.009 